\title{
Temperature dependence of exciton localization dynamics in InxGa1-xN epitaxial films
}

$\operatorname{AUTHOR}(S)$ :

Kanemitsu, Yoshihiko; Tomita, Koichi; Hirano, Daisuke; Inouye, Hideyuki

\section{CITATION:}

Kanemitsu, Yoshihiko ...[et al]. Temperature dependence of exciton localization dynamics in InxGa1-xN epitaxial films. Applied Physics Letters 2006, 88(12): 121113.

\section{ISSUE DATE:}

2006-06

URL:

http://hdl.handle.net/2433/87361

\section{RIGHT:}

c 2006 American Institute of Physics. 


\title{
Temperature dependence of exciton localization dynamics in $\ln _{x} \mathrm{Ga}_{1-x} \mathrm{~N}$ epitaxial films
}

\author{
Yoshihiko Kanemitsu ${ }^{\text {a) }}$ \\ Institute for Chemical Research, Kyoto University, Uji, Kyoto 611-0011, Japan \\ Koichi Tomita \\ Graduate School of Materials Science, \\ Nara Institute of Science and Technology, Ikoma, Nara 630-0192, Japan \\ Daisuke Hirano and Hideyuki Inouye \\ Institute for Chemical Research, Kyoto University, Uji, Kyoto 611-0011, Japan
}

(Received 18 November 2005; accepted 21 February 2006; published online 22 March 2006)

\begin{abstract}
We have studied the temperature dependence of exciton localization dynamics in $\operatorname{In}_{x} \mathrm{Ga}_{1-x} \mathrm{~N}$ epitaxial films $(x=0.09)$ by means of optical Kerr-gate time-resolved photoluminescence (PL) spectral measurements. During 30 ps after 150 fs laser excitation, the PL dynamics is sensitive to the measurement temperature. In the temperature range of 6-50 K, the PL rise time decreases and the PL peak energy shifts to higher energy with an increase of temperature. At high temperatures above $80 \mathrm{~K}$, the thermal quenching of the PL at shallow localized states occurs. The energy relaxation processes of excitons in localized states of $\operatorname{In}_{x} \mathrm{Ga}_{1-x} \mathrm{~N}$ films are discussed. (C) 2006 American Institute of Physics. [DOI: 10.1063/1.2187954]
\end{abstract}

Over the past decade, there have been many experimental and theoretical studies on the optical properties of IIInitride semiconductors such as $\mathrm{GaN}, \mathrm{In}_{x} \mathrm{Ga}_{1-x} \mathrm{~N}$, and $\mathrm{Al}_{y} \mathrm{Ga}_{1-y} \mathrm{~N}$ crystals. ${ }^{1,2}$ Since the fabrication of bright $\mathrm{In}_{x} \mathrm{Ga}_{1-x} \mathrm{~N}$ light-emitting diodes, $\mathrm{In}_{x} \mathrm{Ga}_{1-x} \mathrm{~N}$ ternary alloys are the heart material for optical devices in the blue spectral region. ${ }^{3} \operatorname{In}_{x} \mathrm{Ga}_{1-x} \mathrm{~N}$ ternary alloys show high photoluminescence (PL) quantum efficiencies in spite of high threading dislocation defect density. ${ }^{4}$ It has been reported that the spatial inhomogeneity of indium composition causes the potential fluctuations and efficient PL in $\operatorname{In}_{x} \mathrm{Ga}_{1-x} \mathrm{~N}$ films is due to the radiative recombination of localized excitons. ${ }^{5,6}$ In addition, unusual temperature dependence of the PL spectrum has been observed in $\operatorname{In}_{x} \mathrm{Ga}_{1-x} \mathrm{~N}$ quantum wells and epitaxial layers: The PL peak energy shows complicated behaviors (redshift-blueshift-redshift or blueshift-redshift) with an increase of temperature..$^{7-12}$ This unique temperature dependence of the PL spectrum is also considered to be related to the localization and thermal excitation (delocalization) processes of excitons in $\operatorname{In}_{x} \mathrm{Ga}_{1-x} \mathrm{~N}$ films. Exciton localization in $\mathrm{In}_{x} \mathrm{Ga}_{1-x} \mathrm{~N}$ ternary alloys is one of the most important subjects of fundamental and applied researches. However, the temperature dependence of the localization dynamics of excitons from free state to localized states in $\mathrm{In}_{x} \mathrm{Ga}_{1-x} \mathrm{~N}$ mixed crystals is not clear.

In this letter, we report the PL dynamics of $\operatorname{In}_{x} \mathrm{Ga}_{1-x} \mathrm{~N}$ $(x=0.09)$ epitaxial films at different temperatures by means of an optical Kerr-gate method and a wavelength-tunable femtosecond laser system. It is found that the PL dynamics in the picosecond time region is sensitive to the measurement temperature under resonant excitation of the band edge of $\mathrm{In}_{x} \mathrm{Ga}_{1-x} \mathrm{~N}$. The exciton localization and radiative recombination processes in $\operatorname{In}_{x} \mathrm{Ga}_{1-x} \mathrm{~N}$ will be discussed.

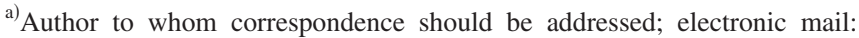
kanemitu@scl.kyoto-u.ac.jp; also at Graduate School of Materials Science, Nara Institute of Science and Technology, Ikoma, Nara 630-0192, Japan.
}

The samples used in this work were fabricated by the metal organic chemical vapor deposition method. ${ }^{13,14}$ The thickness of $\operatorname{In}_{x} \mathrm{Ga}_{1-x} \mathrm{~N}$ epitaxial layers was $90 \mathrm{~nm}$ grown on a patterned sapphire substrate with a 5- $\mu \mathrm{m} \mathrm{GaN}$ buffer layer for the reduction of the nonradiative recombination centers. The $x=0.09$ epitaxial film sample was used in this work. ${ }^{15}$

Wavelength-tunable femtosecond laser pulses were obtained from an optical parametric amplifier system based on a regenerative amplified mode-locked Ti:sapphire laser. The pulse duration and the repetition rate were $\sim 150 \mathrm{fs}$ and $1 \mathrm{kHz}$, respectively. The laser spot size on the sample surface was carefully measured by a knife-edge method. For time-resolved PL spectrum measurements, an optical Kerrgate method was used with toluene as a Kerr medium in a quartz cell. The time resolution of our system was about 0.7 ps. ${ }^{16}$ The PL spectra were measured as a function of the delay time, using a liquid-nitrogen-cooled charged-coupled device $(\mathrm{CCD})$ with a $50-\mathrm{cm}$ single monochromator. In this PL experiment, the excitation photon energy was set to be $\sim 3.136 \mathrm{eV}$, where the excitation photon energy was approximately resonant with the band-gap energy of the $\mathrm{In}_{x} \mathrm{Ga}_{1-x} \mathrm{~N}(x=0.09)$ sample. Under the resonant excitation, the electron temperature just after femtosecond laser excitation is relatively low, and then the PL decay dynamics will be sensitive to the lattice temperature.

Figure 1 shows the temperature dependence of optical absorption and time-integrated PL spectra of the $\operatorname{In}_{x} \mathrm{Ga}_{1-x} \mathrm{~N}$ $(x=0.09)$ epitaxial film sample under weak 150-fs laser excitation at $1.3 \mu \mathrm{J} / \mathrm{cm}^{2}$. The exciton absorption peak and the PL peak in $\operatorname{In}_{x} \mathrm{Ga}_{1-x} \mathrm{~N}$ films are observed at all the measurement temperatures. With an increase of temperature, the PL peak energy slightly shifts to higher energy. At high temperatures above $\sim 80 \mathrm{~K}$, the redshift of the PL energy occurs. There is a large Stokes shift between the absorption and PL peaks at all the measurement temperatures. A large Stokes shift suggests that exciton localization occurs in $\operatorname{In}_{x} \mathrm{Ga}_{1-x} \mathrm{~N}$ films even at high temperatures around $150 \mathrm{~K}$ : The efficient PL is not observed near the free-exciton energy even at high 


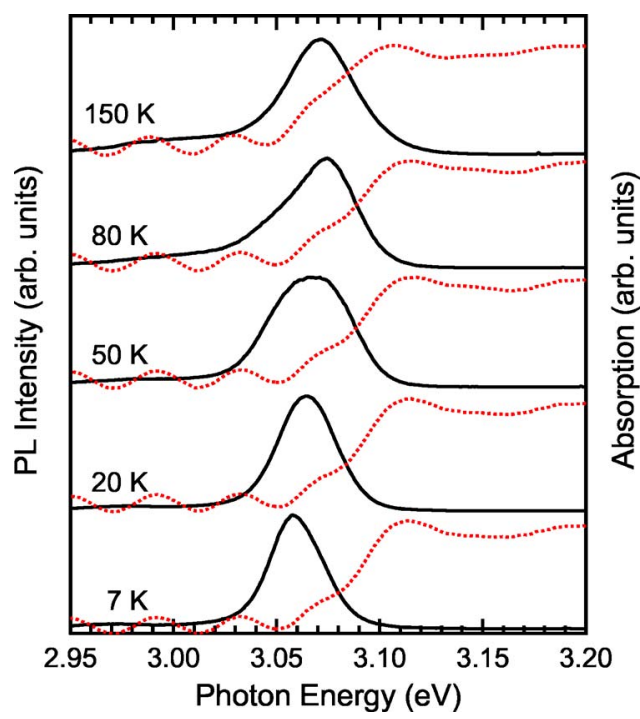

FIG. 1. (Color online) Optical absorption (broken curves) and PL spectra under $1.3 \mu \mathrm{J} / \mathrm{cm}^{2}$ femtosecond laser excitation (solid curves) in the $\operatorname{In}_{x} \mathrm{Ga}_{1-x} \mathrm{~N}$ thin film $(x=0.09)$.

temperatures. The radiative recombination of excitons dominantly occurs after the energy relaxation into the lowest localized state.

In order to clarify the localization process of excitons from the delocalized state to deep localized states, we have measured the PL dynamics of $\operatorname{In}_{x} \mathrm{Ga}_{1-x} \mathrm{~N}$ films as a function of the lattice temperature. The time-resolved PL spectrum directly reflects the population of excitons in the localized states. Figure 2 shows time-resolved PL spectra of the $\mathrm{In}_{x} \mathrm{Ga}_{1-x} \mathrm{~N}$ film $(x=0.09)$ under $230 \mu \mathrm{J} / \mathrm{cm}^{2}$ excitation at different temperatures. A large and rapid redshift of the PL peak energy is clearly observed during $30 \mathrm{ps}$ after the laser excitation. The picosecond PL dynamics is very sensitive to the measurement temperature.

At $6 \mathrm{~K}$, efficient luminescence appears at 5-10 ps after the laser excitation. With an increase of temperature, the luminescence appears at higher energy spectral region and around the zero time delay. For example, the high-energy and fast PL component clearly appears at $50 \mathrm{~K}$, as shown in the figure. This observation shows that the fast radiative recombination of excitons occurs at shallow localized states.

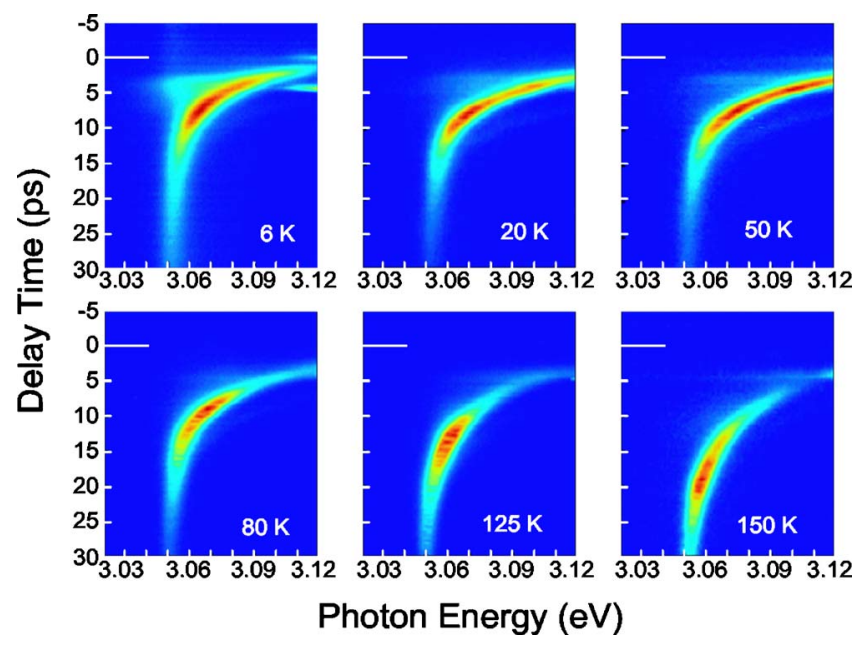

FIG. 2. (Color online) Temperature dependence of the PL dynamics of the

$\mathrm{In}_{x} \mathrm{Ga}_{1-x} \mathrm{~N}$ thin film $(x=0.09)$ under $230 \mu \mathrm{J} / \mathrm{cm}^{2}$ laser excitation. band-edge delocalized state and rapidly relax into localized
Downloaded 16 Nov 2009 to 130.54 .110 .32 . Redistribution subject to AlP license or copyright; see http://apl.aip.org/apl/copyright.jsp
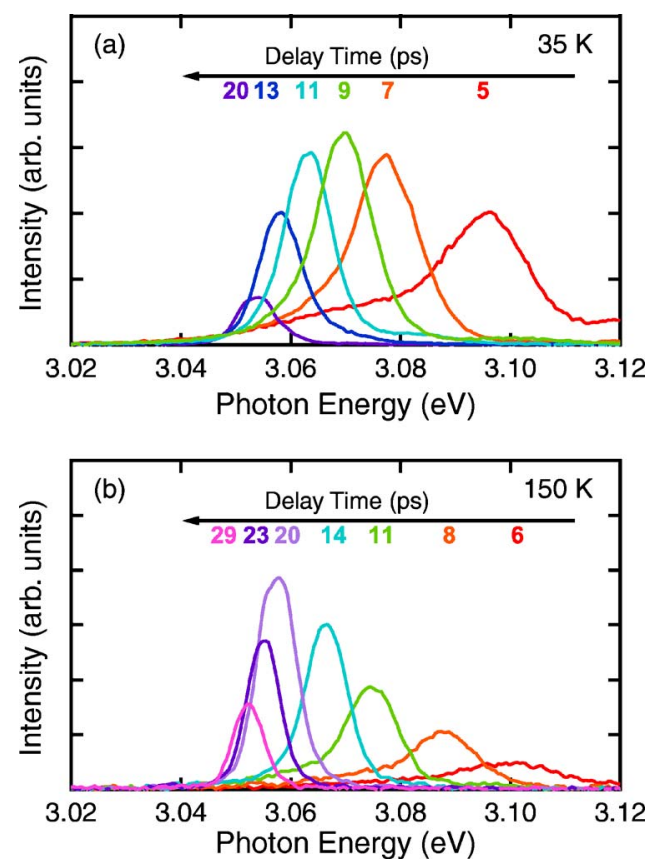

FIG. 3. (Color online) Temporal change of the PL spectrum in the $\mathrm{In}_{x} \mathrm{Ga}_{1-x} \mathrm{~N}$ thin film $(x=0.09)$ at (a) 35 and (b) $150 \mathrm{~K}$.

However, at high temperatures above $80 \mathrm{~K}$, the high-energy and fast PL component disappears. After a large delay time of about 10 ps or more, the PL appears at around the lower localized states. This suggests that at high temperatures the radiative recombination of excitons occurs after the energy relaxation of excitons into deep localized states.

Figure 3 shows, in more detail, PL spectra as a function of the delay time at 35 and $150 \mathrm{~K}$ at $230 \mu \mathrm{J} / \mathrm{cm}^{2}$. At a low temperature of $35 \mathrm{~K}$, a very broad PL appears near the free exciton energy at the 5 ps delay time. With an increase of the delay time, the PL peak energy shifts to lower energy and the PL bandwidth becomes narrower. This behavior can be explained by a picture that radiative excitons relax into the lower energy states and the cooling of the electron temperature occurs during the exciton localization in the band-tail states. There is no significant decrease of the spectrally integrated PL intensity at shallow localized states or at the delay time between 5 and 10 ps.

At a high temperature of $150 \mathrm{~K}$, on the other hand, the PL intensity near the free exciton energy is very weak. The PL intensity increases with a decrease of the energy or with an increase of the delay time: The nonradiative energy relaxation of excitons occurs at shallow localized states and then the efficient radiative recombination of excitons occurs at deep localized states around $3.05 \mathrm{eV}$. The exciton dynamics at shallow delocalized states is very sensitive to the lattice temperature.

Here, we discuss the temperature dependence of the dynamical PL spectrum in the $\operatorname{In}_{x} \mathrm{Ga}_{1-x} \mathrm{~N}$ film $(x=0.09)$. It is believed that the piezoelectric field effect induced by strain does not play a primary role in the observed PL dynamics, because in the $\operatorname{In}_{x} \mathrm{Ga}_{1-x} \mathrm{~N}$ film $(x=0.09)$ the PL spectrum and redshift dynamics depend scarcely on the excitation intensity (Ref. 6) and the redshift time is much shorter than the mean PL lifetime. In semiconductor mixed crystals, the localized states are formed below the edge of the delocalized state. Under the resonant excitation, excitons are generated at the band-edge delocalized state and rapidly relax into localized 
states. The radiative excitons migrate into lower localized states in the $\operatorname{In}_{x} \mathrm{Ga}_{1-x} \mathrm{~N}$ film. It is considered that the observed dynamical redshift of the PL spectrum is determined by the exciton localization processes. At all measurement temperatures between 6 and $150 \mathrm{~K}$, excitons are localized around the lowest energy states within several tens of picoseconds. However, the localization behavior of excitons at localized states between the free-exciton state and the lowest localized states depends strongly on the lattice temperature, as shown in Figs. 2 and 3.

With an increase of temperature from 6 to $50 \mathrm{~K}$, the radiative recombination of excitons occurs at shallow localized states and the continuous PL redshift from the freeexciton states into deep localized states is clearly observed. At elevated temperatures, excitons are thermally excited at shallow localized states around the free-exciton state. At the shallow localized states, the overlap between electron and hole wave functions increases. The radiative recombination rate of excitons at shallow localized states is much larger than that at deep localized states. The population change of excitons in shallow localized states causes the high-energy and fast component in the picosecond PL spectrum, as shown in Fig. 2. Consequently, the radiative excitons at shallow localized state will cause the blueshift of the time-integrated PL spectrum. In fact, the blueshift of the time-integrated PL spectrum occurs with an increase of temperature as shown in Fig. 1. However, the picosecond PL spectrum is very sensitive to the lattice temperature, compared to the case of the time-integrated PL spectrum.

With a further increase of temperature above $80 \mathrm{~K}$, the PL intensity at shallow localized states decreases as shown in Figs. 2 and 3. At high temperatures, the thermal dissociation of excitons occurs at shallow localized states, and electrons and holes are thermally excited into the delocalized states. Thermally excited electrons and holes in the delocalized state are relaxed into deep localized states around the lowest localized state nonradiatively, because at high temperatures the nonradiative relaxation rate is usually much larger than that of radiative recombination of excitons at shallow localized states. Therefore, the PL at the shallow localized states becomes very weak at high temperatures, similar to the case of disordered semiconductors. ${ }^{17}$ On the other hand, at deep localized states, the thermal dissociation of excitons does not occur. The efficient PL appears at around the lowest localized states after a delay time (the relaxation time from the delocalized state to the near-lowest localized state). It is concluded that the energy relaxation process of excitons at shal- low localized states determines the temperature-dependent PL spectrum and dynamics of $\operatorname{In}_{x} \mathrm{Ga}_{1-x} \mathrm{~N}$ films.

In conclusion, we have studied the PL dynamics of $\mathrm{In}_{x} \mathrm{Ga}_{1-x} \mathrm{~N}$ films as a function of temperature under the resonant excitation near the band edge. The picosecond PL dynamics is very sensitive to the lattice temperature. It is shown that the exciton population at shallow localized states determines the unique temperature-sensitive PL spectrum and dynamics in $\operatorname{In}_{x} \mathrm{Ga}_{1-x} \mathrm{~N}$ films. Femtosecond timeresolved PL spectroscopy is one of the most useful methods for understanding the exciton localization dynamics in GaN-based mixed crystals.

The authors thank Professor Y. Yamada of Yamaguchi University for useful discussions. This work was supported in part by the Research Foundation of Opto-Science and Technology and The Futaba Electronics Memorial Foundation.

${ }^{1}$ S. Nakamura, S. Pearton, and G. Fasol, The Blue Laser Diode (Springer, Berlin, 2000).

${ }^{2}$ Properties, Processing and Applications of Gallium Nitride and Related Semiconductors, edited by J. H. Edgar, S. Strite, I. Akasaki, H. Amano, and C. Wetzel (INSPEC, London, 1999).

${ }^{3}$ S. Nakamura, M. Senoh, N. Iwasa, and S. Nagahama, Jpn. J. Appl. Phys., Part 2 34, L797 (1995).

${ }^{4}$ S. Nakamura, Science 281, 956 (1998).

${ }^{5}$ A. Kaneta, T. Mutoh, Y. Kawakami, S. Fujita, G. Murutsuki, Y. Narukawa, and T. Mukai, Appl. Phys. Lett. 83, 3462 (2003), and references therein.

${ }^{6}$ Y. Kanemitsu, K. Tomita, and H. Inouye, Appl. Phys. Lett. 87, 151120 (2005), and references therein.

${ }^{7}$ P. G. Eliseev, P. Perlin, J. Lee, and M. Oslnski, Appl. Phys. Lett. 71, 569 (1997).

${ }^{8}$ Y. H. Cho, G. H. Gainer, A. J. Fischer, J. J. Song, S. Keller, U. K. Mishra, and S. P. DenBaars, Appl. Phys. Lett. 73, 1370 (1998).

${ }^{9}$ K. L. Teo, J. S. Colton, P. Y. Yu, E. R. Weber, M. F. Li, W. Liu, K. Uchida, H. Tokunaga, N. Akutsu, and K. Matsumoto, Appl. Phys. Lett. 73, 1697 (1998).

${ }^{10}$ Y. Narukawa, S. Saijou, Y. Kawakami, S. Fujita, T. Mukai, and S. Nakamura, Appl. Phys. Lett. 74, 558 (1999).

${ }^{11}$ P. Riblet, H. Hirayama, A. Kinosita, A. Hirata, T. Sugano, and Y. Aoyagi, Appl. Phys. Lett. 75, 2241 (1999).

${ }^{12}$ H. P. Schenk, M. Leroux, and P. de Mierry, J. Appl. Phys. 88, 1525 (2000).

${ }^{13}$ C. Sasaki, H. Naito, M. Iwata, H. Kudo, Y. Yamada, T. Taguchi, T. Jyouichi, H. Okagawa, K. Tadatomo, and H. Tanaka, J. Appl. Phys. 93, 1642 (2003).

${ }^{14}$ K. Tadatomo, H. Okagawa, Y. Ohuchi, T. Tsunekawa, Y. Imada, M. Kato, and T. Taguchi, Jpn. J. Appl. Phys., Part 2 40, L583 (2001).

${ }^{15} \mathrm{Thick} \mathrm{In}_{x} \mathrm{Ga}_{1-x} \mathrm{~N}$ epitaxial films were used to reduce piezoelectric field effects and the inhomogeneous PL broadening due to the fluctuation of the layer thickness in the quantum well structures.

${ }^{16}$ T. Nagai, A. Yamamoto, and Y. Kanemitsu, Phys. Rev. B 71, 121201(R) (2005).

${ }^{17}$ Y. Kanemitsu and T. Kushida, Appl. Phys. Lett. 77, 3550 (2000). 